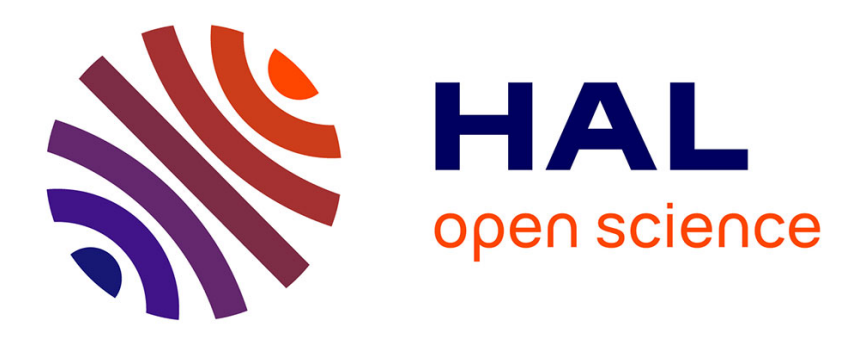

\title{
Peierls-Nabarro landscape for martensitic phase transitions
}

Lev Truskinowsky, Anna Vainchtein

\section{To cite this version:}

Lev Truskinowsky, Anna Vainchtein. Peierls-Nabarro landscape for martensitic phase transitions. Physical Review B: Condensed Matter and Materials Physics (1998-2015), 2003, 67 (17), 10.1103/physrevb.67.172103 . hal-00111393

\section{HAL Id: hal-00111393 \\ https://hal.science/hal-00111393}

Submitted on 29 Jul 2019

HAL is a multi-disciplinary open access archive for the deposit and dissemination of scientific research documents, whether they are published or not. The documents may come from teaching and research institutions in France or abroad, or from public or private research centers.
L'archive ouverte pluridisciplinaire $\mathbf{H A L}$, est destinée au dépôt et à la diffusion de documents scientifiques de niveau recherche, publiés ou non, émanant des établissements d'enseignement et de recherche français ou étrangers, des laboratoires publics ou privés. 


\title{
Peierls-Nabarro landscape for martensitic phase transitions
}

\author{
Lev Truskinovsky* \\ Laboratoire de Mechanique des Solides, CNRS-UMR 7649, Ecole Polytechnique, 91128 Palaiseau, France
}

Anna Vainchtein ${ }^{\dagger}$

Department of Mathematics, University of Pittsburgh, Pittsburgh, Pennsylvania 15260, USA

(Received 6 December 2002; published 19 May 2003)

\begin{abstract}
We consider a prototypical model of a martensitic phase transition, which takes discreteness and nonlocality into account and allows one to reconstruct explicitly the Peierls-Nabarro energy landscape for the phase boundaries. We solve the discrete problem exactly and obtain a simple expression for the critical Peierls force that is necessary to release a lattice-trapped phase boundary. The model suggests specific relations between the microscopic parameters of the lattice and the size of the hysteresis in martensitic materials, which we verify for the cubic- $\beta_{1}^{\prime}$-monoclinic phase transition in $\mathrm{Cu}-\mathrm{Al}-\mathrm{Ni}$ alloy.
\end{abstract}

DOI: $10.1103 /$ PhysRevB.67.172103

PACS number(s): 64.70.Kb, 61.72.Bb, 63.90.+t

Rate-independent hysteresis is commonly observed in materials undergoing martensitic phase transitions, e.g., Ag-Cd, ${ }^{1}$ $\mathrm{In}-\mathrm{Tl},{ }^{2} \mathrm{Cu}-\mathrm{Al}-\mathrm{Ni},{ }^{3,4}$ and $\mathrm{Ni}-\mathrm{Ti}^{5,6}$ When subjected to a quasistatic loading, these materials deform elastically until the load reaches a threshold when one or several phase boundaries nucleate and propagate through the specimen; upon unloading, the reverse transformation takes place at a lower critical load. ${ }^{7,8}$ While it is understood that the resulting rateindependent hysteresis reflects the presence of multiple metastable configurations, the physical origin of the upper and lower critical loads has been a subject of active debate emphasizing factors such as disorder, ${ }^{9}$ elastic incompatibility, ${ }^{10}$ and surface energy. ${ }^{11}$ The goal of the present paper and of its forthcoming dynamic extension ${ }^{12}$ is to explore the effects of material discreteness as a source of the overall ruggedness of the energy landscape and of the associated hysteresis. By following the pattern of plasticity theory, we relate hysteresis to the lattice trapping of phase boundaries viewed as the main carriers of inelastic deformation.

We recall that in plasticity theory, the yield stress is related to the Peierls force ${ }^{13,14}$ representing a threshold needed to release trapped dislocations and set plastic deformation in motion. The dislocations in this approach are viewed as moving in a Peierls-Nabarro $(\mathrm{PN})$ periodic potential with the valleys representing pinned equilibrium configurations. The applied shear stress tilts the PN landscape until at the critical level (Peierls force), characterizing the yield strength of the crystal, the barriers between the valleys completely disappear.

Similar to dislocations, martensitic phase boundaries represent highly mobile planar defects whose resonant interaction with a crystal lattice leads to an intense radiative damping and results in dry-friction-type kinetics. ${ }^{15}$ The goal of the present paper is to develop for martensitic phase boundaries a prototypical one-dimensional model capturing the effect of lattice trapping and allowing for a transparent analytical study of both statics and dynamics. As a first step, we explicitly reconstruct the fine structure of the PN landscape for the proposed model and relate the critical Peierls force to the microparameters of the model characterizing interatomic interactions.
The simplest physical description of a stress-driven martensitic phase transition in lattices can be obtained from the study of a chain of bistable elements. ${ }^{16-19}$ The same model may also represent a collection of rigid atomic planes interacting through shear snap springs. To mimic the threedimensional nature of the actual problem, it is natural to complement the anharmonic nearest-neighbor (NN) interactions by a harmonic interaction of the next-to-nearest neighbors (NNN). The resulting potential energy, which represents a simplified version of a nonlocal discrete model studied numerically in Ref. 20, can be written in the form

$$
\Psi=\varepsilon \sum_{k=-\infty}^{\infty}\left[\phi\left(\frac{u_{k}-u_{k-1}}{\varepsilon}\right)+\frac{\gamma}{2}\left(\frac{u_{k+1}-u_{k-1}}{\varepsilon}\right)^{2}\right] .
$$

Here, $u_{k}$ is the displacement of the $k$ th particle with respect to the homogeneous reference configuration with spacing $\varepsilon$. The first term in the sum represents the double-well energy of the NN interactions, the second term is the energy of the linear NNN interactions, characterized by the modulus $\gamma$. Some numerical experiments aimed at locating the global energy minima for a finite discrete system similar to (1) can be found in Refs. 21 and 22; in continuum limit, the ground state for this class of energies was studied in Refs. 22 and 23. We remark that in terms of strains $w_{k}=\left(u_{k}-u_{k-1}\right) / \varepsilon$, energy (1) can be rewritten as the energy of the FrenkelKontorova (FK) model: ${ }^{24}$

$$
\Psi=\varepsilon \sum_{k=-\infty}^{\infty}\left[\widetilde{\phi}\left(w_{k}\right)-\frac{\gamma}{2}\left(w_{k}-w_{k-1}\right)^{2}\right],
$$

where $\widetilde{\phi}\left(w_{k}\right)=\phi\left(w_{k}\right)+4 \gamma w_{k}^{2}$. Although in statics the two models are equivalent, their dynamic extensions are entirely different.

Since our aim is to obtain analytical results, we further simplify the model by assuming that the NN potential $\phi(w)$ is biparabolic with a piecewise linear derivative

$$
\phi^{\prime}(w)=K\left(w-a \theta\left(w-w_{c}\right)\right)
$$

where $\theta(x)$ is the Heaviside function. In this case the spinodal region reduces to a point, so that the NN springs are in 
phase I if the strain $w$ is below the critical value $w_{c}$ and in phase II otherwise. Other parameters of the potential $\phi(w)$ are the transformation strain $a$ and the elastic modulus $K$ $>0$, which we assume to be identical in both phases. The elastic modulus $\gamma$ of the NNN springs is taken to be negative, as suggested, for instance, by molecular models with the Lennard-Jones potential. ${ }^{25}$ In what follows we focus on the representation of the PN landscape associated with the model (1) and (3); a related analysis for the piecewise linear FK model at zero driving and limited to thecomputation of the Peierls barrier can be found in Refs. 26 and 27.

We begin with the localization of the valleys of the PN potential representing equilibrium configurations of Eqs. (1) and (3) with a single interface. From the expression of the energy, we obtain the following equilibrium equations:

$$
A_{k l} w_{l}=p\left(w_{k}\right) .
$$

Here, the tridiagonal symmetric matrix $A_{k l}$, which describes the "elasticity" of the lattice with NNN interactions, is defined by $A_{k l} w_{l}=w_{k}+\{(\eta-1) / 4\}\left(w_{k+1}+2 w_{k}+w_{k-1}\right)$, where $\eta=(K+4 \gamma) / K$ is the main nondimensional parameter of the problem measuring the relative strength of the NN and NNN interactions. The loading function $p(w)=F / K$ $+a \theta\left(w-w_{c}\right)$ contains the constant driving $F$ representing, for instance, the applied shear stress.

The trivial solution of the equilibrium equations (4) corresponds to a uniform deformation in either of the phases. Thus, the chain is in phase I with constant $w_{k}=F / K \eta$ when $F \leqslant K \eta w_{c}$. We assume that at the given loading, this homogeneous configuration is stable, which in our case $(K>0$, $\gamma<0)$ translates into the requirement that $0<\eta<1 .{ }^{25}$ To find a nontrivial solution of Eq. (4) describing an isolated phase boundary pinned by the lattice, we assume that the location of the interface coincides with particle $i$. This leads to the constraints $w_{k} \geqslant w_{c}, k \leqslant i$ (phase II) and $w_{k} \leqslant w_{c}, k$ $>i$ (phase $\mathrm{I}$ ). The system of nonlinear equilibrium equations (4) can then be rewritten as a system of linear equations with a given right-hand side:

$$
p\left(w_{k}\right)=F / K+a \theta(i-k) .
$$

Solutions of Eq. (5) must tend to the homogeneous configurations $w_{-\infty}=(F / K+a) / \eta$ and $w_{+\infty}=F /(K \eta)$ at infinity. The exact solution of this linear problem is given by

$$
w_{k}(i)=w^{*}+s(k-i),
$$

where

$$
w^{*}=\frac{F}{E}, \quad s(m)=\Delta\left\{\theta\left(\frac{1}{2}-m\right)-\frac{e^{-|m-1 / 2| \lambda}}{2 \cosh (\lambda / 2)}\right\} \operatorname{sgn}\left(\frac{1}{2}-m\right),
$$

and $\lambda=2 \operatorname{arccosh}(1 / \sqrt{|\eta-1|})$. Parameters $E=K \eta$ and $\Delta$ $=a / \eta$ define the macroscopic elastic modulus and the transformation strain, respectively, for the homogeneously deformed system with the macroscopic response $F=E(w$ $\left.-\Delta \theta\left(w-w_{c}\right)\right)$.

Using Eq. (6), one can show that the strains in the phases are confined to the corresponding energy wells if and only if the applied force lies in the interval $F_{M}-F_{P} \leqslant F \leqslant F_{M}$
$+F_{P}$. Here, $F_{M}=E\left(w_{c}-\Delta / 2\right)$ is the equilibrium (Maxwell) force for the homogeneous system defined by the corresponding equal area construction, and

$$
F_{P}=E \Delta \sqrt{\eta} / 2
$$

is the Peierls force. One can see that after the strain in the $(i+1)$ th NN spring reaches the critical value $w_{c}$ at $F=F_{M}$ $+F_{P}$, the single interface solution ceases to exist, making the phase boundary free to move. During the unloading, the depinned phase boundary starts moving in the opposite direction once the strain in the $i$ th reaches the value $w_{c}$ at $F$ $=F_{M}-F_{P}$. Notice that inside the interval of lattice trapping $F_{M}-F_{P}<F<F_{M}+F_{P}$, solution (6) is at least metastable due to the positive definiteness of the matrix $A_{k l}$, guaranteed by our assumptions on elastic moduli.

To reconstruct the full PN energy landscape, one needs to find the optimal (minimal barrier) path connecting the metastable equilibria of a single interface. Assume for determinacy that $F_{M}<F<F_{P}+F_{M}$ and consider the two adjacent pinned equilibrium configurations $w_{k}(i)$ and $w_{k}(i+1)$. Notice that in the first of these configurations the $(i+1)$ th spring is still in phase I, while in the second this spring has already switched to phase II. In order to move from the first to the second configuration, the system has to follow a nonequilibrium path with $(i+1)$ th spring changing phase. We may therefore choose $w_{i+1}$ as the order parameter and minimize the energy with respect to all $w_{k}$, with $k \leqslant i$ and $k \geqslant i$ +2 . The necessary conditions for this constrained equilibrium are given by equations (4), with $k \neq i+1$. For $k \leqslant i$, the solution of the resulting linear system satisfying the boundary condition at $-\infty$ can be written in the parametric form

$$
w_{k}^{-}(\nu)=w^{*}+\Delta\left\{1-\frac{e^{\lambda(k-\nu-1 / 2)}}{2 \cosh (\lambda / 2)}\right\}
$$

Here, $\nu$ is defined by the condition $w_{i+1}^{-}(\nu)=w_{i+1}$; it is easy to see that $i=[\nu]$ (the integer part of $\nu$ ). In the interval $(i, i+1)$, the function $w_{i+1}^{-}(\nu)$ monotonically increases with $\nu$ from $w_{i+1}(i)$ to $w_{i+1}(i+1)$ and $\nu$ can be used as a new order parameter. To complete the construction of the constrained equilibria, we need to find $w_{k}^{+}(\nu)$ satisfying equilibrium equations for $k \geqslant i+2$, the boundary condition at $+\infty$ and the continuity condition $w_{i+1}^{+}(\nu)=w_{i+1}^{-}(\nu)$. We obtain

$$
w_{k}^{+}(\nu)=w^{*}+\Delta e^{\lambda([\nu]+1-k)}\left\{1-\frac{e^{\lambda([\nu]-\nu+1 / 2)}}{2 \cosh (\lambda / 2)}\right\} .
$$

The combination of $w_{k}^{-}(\nu)$ taken at $k \leqslant[\nu]+1$ and $w_{k}^{+}(\nu)$ at $k \geqslant[\nu]+1$ constitutes the desired nonequilibrium transformation path $w_{k}(\nu)$. Notice that by construction, the integer values of $\nu$ correspond to the metastable configurations of the pinned phase boundary with the strain profile $w_{k}(i)$.

To obtain the associated PN landscape, we introduce the Gibbs energy $G=\Psi-F \sum_{k=-\infty}^{\infty} w_{k}$ and compute its values along the nonequilibrium paths (8) and (9). Since the energy of the chain is infinite, we need to evaluate the difference between $G(\nu)$ and the energy of one of the metastable equi- 


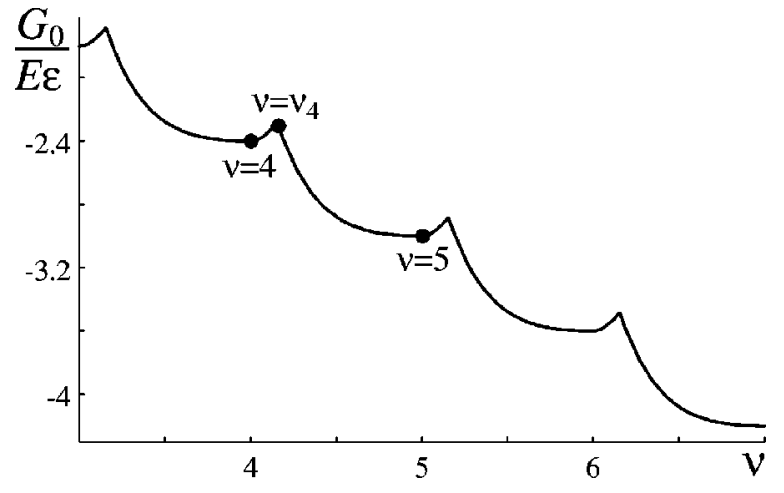

FIG. 1. Peierls-Nabarro energy landscape $G_{0}(\nu) /(E \varepsilon)$ at $F / E$ $=0.3, \eta=1 / 2, w_{c}=a=1$.

libria. Defining $G_{0}(\nu)=G(\nu)-G(0)$ and substituting Eqs. (8) and (9) into Eq. (1), we obtain

$$
\begin{aligned}
G_{0}(\nu)= & -E \Delta \varepsilon\left\{\frac{\Delta(\eta-1) \sqrt{\eta}}{8}\left(1-e^{-\lambda(\nu-[\nu])}\right)^{2}\right. \\
& \left.+\frac{\bar{F}[\nu]}{E}+h(\nu) \theta(h(\nu))\right\} .
\end{aligned}
$$

Here, $\bar{F}=F-F_{M}$ is the difference between the applied and Maxwell forces, and $h(\nu)=\bar{F} / E+(\Delta / 2)\{1$ $\left.-e^{-\lambda(\nu-[\nu]-1 / 2)} / \cosh (\lambda / 2)\right\}$.

A typical graph of $G_{0}(\nu)$ is shown in Fig. 1. As expected, the PN landscape exhibits local minima at integer values of $\nu$ corresponding to successive metastable pinned locations of the phase boundary. In order to switch from one pinned configuration (say, at $\nu=i$ ) to the neighboring one (at $\nu=i$ $+1)$, the system needs to climb an energy barrier. In the present piecewise linear setting, the maxima of the PN potential (saddle points of the original energy) are represented by the sharp peaks. ${ }^{28}$ For each $i$, the peak is located at $\nu_{i}$ $\in(i, i+1)$, which is defined by the condition $w_{i+1}^{ \pm}\left(\nu_{i}\right)$ $=w_{c}$. The energy barrier associated with a peak does not depend on $i$ and is given by the following explicit formula

$$
G_{0}\left(\nu_{i}\right)-G_{0}(i)=\frac{\left(F_{P}-\bar{F}\right)^{2} \varepsilon \Delta}{4 F_{P}} .
$$

The barrier is maximal at Maxwell force $(\bar{F}=0)$ and vanishes at the upper boundary of the trapping region where $\bar{F}$ $=F_{P}$. If the energy $\phi(w)$ contains a nondegenerate spinodal region, the cusps are replaced by the smooth energy maxima ${ }^{18}$ but the $\gamma$ dependence of the Peierls barrier becomes much more complex. ${ }^{24}$

A parameter playing an important role in the formulation of the kinetic relations for phase boundaries ${ }^{15}$ is the driving force $g$ represented in the three-dimensional continuum setting by the jump of the normal component of the Eshelby tensor. $^{29}$ In our framework, $g=\left\{G_{0}(i)-G_{0}(i+1)\right\} / \varepsilon$ $=\bar{F} \Delta$, which is again independent of $i$. One can see that $g$ characterizes the degree of tilting of the PN landscape due to the external force $F$. In particular, the driving force equals zero at $\bar{F}=0$ (horizontal PN landscape) and reaches its maximum at $\bar{F}=F_{P}$ when the barriers disappear. The critical driving force is equal to

$$
g_{P}=F_{P} \Delta,
$$

where the Peierls force is given by Eq. (7).

In the limiting case $\eta=1$ when NNN interactions are absent, the PN landscape reduces to the expression computed in Ref. 18, and the limiting minimal barrier path corresponds to a single spring changing phase, with other springs remaining fixed in their equilibrium positions. We remark, however, that in the NN chain the location of the spring that changes phase is arbitrary. When NNN interactions are taken into account, the permutational degeneracy is removed and the transforming spring is located exactly in front of the phase boundary. The existence of the boundary layer in the NNN chain makes the propagation of the existing phase boundary easier than the nucleation of a new one, which has also been noticed in the numerical experiments conducted for the finite chains in Ref. 20. In the present model, this is manifested by the fact that the Peierls force $F_{P}$ associated with the propagation of a phase boundary is smaller than the spinodal force $F_{S}=E \Delta / 2$ marking the threshold of instability for the homogeneous state. In the local model without NNN interactions the corresponding "nucleation peak" is absent and $F_{P}=F_{S} \cdot 18$

To verify the theory, we estimate the modulus of NNN interactions using the experimental data on kinetics of martensitic phase boundaries and then compare it with an independent estimate based on a simple molecular model of the same solid phase. Formula (7) for the Peierls force implies that

$$
\eta=\left\{1+2\left(F_{P}-F_{S}\right) /(E \Delta)\right\}^{2} .
$$

Then, if parameters $F_{P}, E$, and $\Delta$ are known from the macroscopic experiment, one can determine parameter $\eta$ from Eq. (13). Note, however, that the assumption about the bilinear character of the force-strain curve for a single spring largely overestimates the maximal hysteresis width. ${ }^{4}$ While a more appropriate estimate could, in principle, be obtained from the model with the cubic force-strain relation, the associated discrete model does not lend itself to an analytical study. To obtain an improved estimate of $\eta$, we assume that in the realistic case when $F_{P}$ is close to $F_{S},{ }^{20}$ the linearized version of Eq. (13),

$$
\eta \approx 1+4\left(F_{P}-F_{S}\right) /(E \Delta)
$$

remains true for the cubic model with the same $E \Delta$. For the cubic interpolation of Eq. (3), the spinodal force reduces to $F_{S}=E \Delta /(6 \sqrt{3})$. The Peierls force $F_{P}$ can then be found from formula (12) relating it to the critical driving force $g_{P}$, which, in turn, can be obtained from the data on kinetics of martensitic phase boundaries.

To estimate $\eta$, we use the critical driving force $g_{P}$ $=25.26 \mathrm{MPa}$ reported in Ref. 30 for a moving interface between cubic and $\beta_{1}^{\prime}$-monoclinic phases in a single-crystal $\mathrm{Cu}-\mathrm{Al}-\mathrm{Ni}$ alloy. The other relevant parameters, also provided 
in Ref. 30, are $\Delta=0.16868$ and $E=10 \mathrm{GPa}$. Substituting these values into Eqs. (12) and (14), we obtain $\eta=0.97$, which corresponds to $\gamma=-77.13 \mathrm{MPa}$ and $K=10.3 \mathrm{GPa}$.

To check this result, we can alternatively estimate $\eta$ by assuming that both $\mathrm{NN}$ and $\mathrm{NNN}$ interactions are governed by the Lennard-Jones potential $U(r)=(K \varepsilon / 72)\left\{(\varepsilon / r)^{12}\right.$ $\left.-2(\varepsilon / r)^{6}\right\}$, where parameters are selected so that elastic modulus in the potential well located at $r=\varepsilon$ equals $K$. By linearizing $U(r)$ around the unstretched homogeneous state with the spacings $r=\varepsilon$ and $r=2 \varepsilon$, we obtain ${ }^{25} \eta$ $=4 U^{\prime \prime}(2 \varepsilon) / U^{\prime \prime}(\varepsilon)$. This yields $\eta=0.98$, which, despite a rather rigid form of the potential, is remarkably close to the above estimate. With macroscopic elastic modulus $E$ $=10 \mathrm{GPa}$, we obtain $\gamma=-45.1 \mathrm{MPa}$ and $K=10.18 \mathrm{GPa}$.
We conclude that despite its simplicity, the proposed model provides a realistic link between the macroscopic hysteresis and the microscopic parameters of interatomic interactions. To study the behavior of the driven system inside the hysteresis loop, one needs to analyze the dynamic extension of this model. Our preliminary results show that the ruggedness of the PN landscape exposed in the present paper gives rise to stick-slip motion of the phase boundaries at small averaged velocities and nonzero radiative drag at subsonic and near sonic velocities.

The authors would like to thank A. Vainshtein for his helpful remarks. This research was supported by NSF grants DMS-0102841 (L.T.) and DMS-0137634 (A.V.).
*Electronic address: trusk@1ms.polytechnique.fr

†Electronic address: aav4@pitt.edu

${ }^{1}$ R. Krishnan, Mater. Sci. Forum 3, 387 (1985).

${ }^{2}$ M.W. Burkart and T.A. Read, Trans. AIME 197, 1516 (1953).

${ }^{3}$ T.W. Shield, J. Mech. Phys. Solids 43, 869 (1995).

${ }^{4}$ K. Otsuka, H. Sakamoto, and K. Shimizu, Acta Metall. 27, 585 (1979).

${ }^{5}$ J.A. Shaw and S. Kyriakides, Acta Mater. 45, 683 (1997).

${ }^{6}$ P.H. Leo, T.W. Shield, and O.P. Bruno, Acta Metall. Mater. 41, 2477 (1993).

${ }^{7}$ Shape Memory Materials, edited by K. Otsuka, K. Shimizu, M. Doyama, S. Somiya, and R.P.H. Chang (Materials Research Society, Pittsburgh, 1989).

${ }^{8}$ Proceedings of the Workshop on First-Order Displacive Phase Transformations, edited by L.E. Tanner and M. Wuttig [Mater. Sci. Eng., A 127, 137 (1990)].

${ }^{9}$ J.P. Sethna, et al., Phys. Rev. Lett. 70, 3347 (1993).

${ }^{10}$ R. Abeyaratne, C. Chu, and R.D. James, Philos. Mag. A 73, 457 (1996).

${ }^{11}$ L. Truskinovsky and G. Zanzotto, J. Mech. Phys. Solids 44, 1371 (1996).

${ }^{12}$ L. Truskinovsky and A. Vainchtein (unpublished).

${ }^{13}$ F.R.N. Nabarro, Theory of Crystal Dislocations (Dover, New York, 1987).

${ }^{14}$ R.N. Peierls, Proc. Phys. Soc. London 52, 34 (1940).

${ }^{15}$ O. Kresse and L. Truskinovsky, J. Mech. Phys. Solids 51, 1305 (2003).
${ }^{16}$ I. Müller and P. Villaggio, Arch. Ration. Mech. Anal. 65, 25 (1977).

${ }^{17}$ B. Fedelich and G. Zanzotto, J. Nonlinear Sci. 2, 319 (1992).

${ }^{18}$ G. Puglisi and L. Truskinovsky, J. Mech. Phys. Solids 50, 165 (2002).

${ }^{19}$ G. Puglisi and L. Truskinovsky, Continuum Mech. Thermodyn. 14, 437 (2002).

${ }^{20}$ Y.Y. Ye, C.T. Chan, and K.M. Ho, Phys. Rev. Lett. 66, 2018 (1991).

${ }^{21}$ N. Triantafyllidis and S. Bardenhagen, J. Elast. 33, 259 (1993).

${ }^{22}$ S. Pagano and S. Paroni, Q. Appl. Math. 61, 89 (2003).

${ }^{23}$ A. Braides and M.S. Gelli, J. Convex Anal. 9, 363 (2002).

${ }^{24}$ O. Braun and Y. Kivshar, Phys. Rep. 306, 1 (1998).

${ }^{25}$ M. Charlotte and L. Truskinovsky, J. Mech. Phys. Solids 50, 217 (2002).

${ }^{26}$ J.H. Weiner and W.T. Sanders, Phys. Rev. 134, A1007 (1964).

${ }^{27}$ J. Kratovchil and V.I. Indenbom, Czech. J. Phys., Sect. B 13, 814 (1963).

${ }^{28}$ Observe that as a result of nonsmoothness of our energy, the $i$ th and $(i+1)$ th particles are not in equilibrium at a saddle point; instead, they are subjected to forces of equal magnitude $R=\left(F_{P}-\bar{F}\right) / \sqrt{\eta}$ and opposite directions. For an analogous result in the FK model, see R. Hobart, J. Appl. Phys. 36, 1944 (1965).

${ }^{29}$ L. Truskinovsky, J. Appl. Math. Mech. (PMM) 51, 777 (1987).

${ }^{30}$ R. Abeyaratne and J.K. Knowles, Acta Mater. 45, 1671 (1997). 